\title{
Supplementation with EPA or fish oil for 11 months lowers circulating lipids, but does not delay the onset of diabetes in UC Davis-type 2 diabetes mellitus rats
}

\author{
Bethany P. Cummings ${ }^{1,2}$, Kimber L. Stanhope ${ }^{1,2}$, James L. Graham ${ }^{1,2}$, Steven C. Griffen ${ }^{3}$ and \\ Peter J. Havel ${ }^{1,2 *}$ \\ ${ }^{1}$ Department of Molecular Biosciences, School of Veterinary Medicine, University of California, Davis, One Shields Avenue, Davis, \\ CA 95616, USA \\ ${ }^{2}$ Department of Nutrition, University of California, Davis, Davis, CA, USA \\ ${ }^{3}$ Department of Internal Medicine, University of California, Davis, Sacramento, CA, USA
}

(Received 20 January 2010 - Revised 28 May 2010 - Accepted 1 June 2010 - First published online 24 August 2010)

EPA or fish oil supplementation has been suggested as treatments for the prevention of type 2 diabetes mellitus (T2DM) due to their lipid-lowering and potential insulin-sensitising effects. We investigated the effects of supplementation with EPA ( $1 \mathrm{~g} / \mathrm{kg}$ body weight per $\mathrm{d})$ or fish oil $(3 \mathrm{~g} / \mathrm{kg}$ body weight per d) on the age of onset of T2DM and circulating glucose, insulin, lipids, leptin and adiponectin in UC Davis (UCD)-T2DM rats. Animals were divided into three groups starting at 1 month of age: control, EPA and fish oil. All the animals were followed until diabetes onset or for up to 12 months of age. Monthly fasting blood samples were collected for the measurement of glucose, lipids, hormones and C-reactive protein (CRP). Neither EPA nor fish oil delayed the onset of T2DM or altered fasting plasma glucose, insulin, CRP, adiponectin or leptin concentrations. The groups did not differ in energy intake or body weight. Fish oil treatment lowered fasting plasma TAG concentrations by 39 (SD 7) \% $(P<0.001)$ and EPA lowered fasting plasma NEFA concentrations by $23(\mathrm{SD} 5) \%(P<0.05)$ at 4 months of age compared with the control group. EPA and fish oil lowered fasting plasma cholesterol concentrations at 4 months of age by 19 (SD 4) and 22 (SD 4 ) \% compared with the control group, respectively (both $P<0 \cdot 01$ ). In conclusion, EPA and fish oil supplementation lowers circulating lipid concentrations, but does not delay the onset of T2DM in UCD-T2DM rats.

Diabetes prevention: EPA: Fish oil: TAG

Consumption of EPA and fish oil (consisting of EPA and DHA) has been reported to reduce inflammation ${ }^{(1-5)}$ and improve cardiovascular risk factors ${ }^{(6-9)}$, and possibly improve insulin sensitivity ${ }^{(10,11)}$. These effects suggest that chronic supplementation with EPA and fish oil might potentially delay or prevent the onset of type 2 diabetes mellitus (T2DM).

Many of the beneficial effects attributed to EPA and fish oil intake, including the potential to improve insulin sensitivity, appear to be related to a reduction of inflammation via two main pathways. First, chronic consumption of fish oil and EPA results in greater membrane phospholipid EPA content at the expense of arachidonic acid ${ }^{(1)}$. Arachidonic acid is the precursor to multiple inflammatory eicosanoid products, whereas EPA is a poor substrate for eicosanoid production, and EPAderived eicosanoids are generally less bioactive ${ }^{(12)}$. Secondly, EPA has been shown to activate PPAR-mediated suppression of NF- $\mathrm{KB}$, a potent activator of inflammatory mediators ${ }^{(13-15)}$. Inflammation is believed to play a crucial role in the onset and progression of T2DM, as expanding adipocytes secrete increasing amounts of inflammatory mediators, such as IL-6 which stimulates hepatic C-reactive protein (CRP) release, potentially impairing insulin signalling ${ }^{(16,17)}$.

In addition to their impact on inflammation, EPA and fish oil consumption has been reported to lower circulating lipids in rodents and human subjects ${ }^{(6,7,11,18)}$. This action is mediated in part by activation of PPAR- $\alpha$ (the pharmaceutical target for fibrates) and $\gamma$ (the pharmaceutical target for thiazolidinediones). Activation of PPAR- $\gamma$ by EPA and fish oil-derived fatty acids has been shown to increase secretion of the adipocyte hormone, adiponectin ${ }^{(3,5,11,19,20)}$. Adiponectin promotes improvement of insulin sensitivity and has potential lipid-lowering effects, in part by activating adenosine monophosphate-activated protein kinase and increasing fatty acid oxidation ${ }^{(19)}$. PPAR- $\gamma$ agonists may also act to lower circulating lipids by expanding subcutaneous adipose depots for fatty acid storage ${ }^{(21)}$.

While the cardioprotective effects of marine-derived lipids as nutritional supplements are becoming increasingly clear, no studies have addressed the efficacy of EPA or fish oil in the prevention T2DM. Some studies in rodent models have 
demonstrated improved insulin sensitivity during fish oil treatment ${ }^{(11,22)}$; however, other studies in rodents ${ }^{(23,24)}$ and clinical studies in healthy subjects and patients with T2DM have demonstrated that neither EPA nor fish oil improves insulin sensitivity or lower plasma glucose $^{(4,7,24-27)}$. Therefore, we sought to determine whether EPA or fish oil can delay or prevent the onset of diabetes and/or lower circulating lipids and markers of inflammation in UC Davis (UCD)-T2DM rats, a rat model of T2DM with polygenic adult-onset obesity and insulin resistance, and inadequate $\beta$-cell compensation ${ }^{(28)}$. Furthermore, we sought to determine whether there are differences between the chronic effects of EPA and fish oil supplementation by examining the two treatment groups in parallel and providing the same amount of $n-3$ fatty acids (as EPA or as EPA + DHA) in both groups.

\section{Methods}

Animals and diets

The UCD-T2DM rat model was produced by crossing obese Sprague-Dawley rats prone to adult-onset obesity and insulin resistance $^{(29,30)}$ with Zucker diabetic fatty lean rats that have intact leptin signalling, but a defect in pancreatic $\beta$-cell insulin gene transcription ${ }^{(31)}$. This cross resulted in a new rat model that develops polygenic adult-onset obesity and diabetes in both sexes with rats exhibiting insulin resistance, impaired glucose tolerance and eventual $\beta$-cell decompensation ${ }^{(28)}$. These rats develop diabetes when fed a standard low-fat rodent chow diet and also demonstrate a later age of diabetes onset than other rodent models of T2DM, such as the Zucker diabetic fatty rat, making them highly suitable for diabetes prevention studies ${ }^{(28,32)}$.

Rats were housed in hanging wire cages in the animal facility in the Department of Nutrition at the University of California, Davis, and maintained on a $12 \mathrm{~h}$ light-dark cycle. Food intake and body weight were measured three times a week. Non-fasting blood glucose was monitored every week with a glucose meter (One-Touch Ultra, LifeScan, Milpitas, CA, USA) at 13.00-14.00 hours. Diabetes onset was defined as a non-fasted blood glucose value above $11.1 \mathrm{mmol} / \mathrm{l}$ $(2000 \mathrm{mg} / \mathrm{l})$ on two consecutive weeks ${ }^{(26)}$. All rats were followed until 1 year of age or for 4 months after the onset of diabetes. The experimental protocols were approved by the University of California, Davis Institutional Animal Care and Use Committee.

Starting at 1 month of age, male animals were divided into three groups: control ( $n$ 20), EPA ( $n$ 15) and fish oil ( $n$ 17). Purified ethyl-all-cis-5,8,11,14,17-icosapentaenoate (Mochida Pharmaceutical Company, Shizuoka, Japan) or fish oil (18\% EPA, $12 \%$ DHA; Jedwards, Quincy, MA, USA) was mixed with ground rodent chow (no. 5012; PMI Nutrition International LLC, Brentwood, MO, USA) to provide a dose of $1 \mathrm{~g} \mathrm{EPA} / \mathrm{kg}$ body weight per $\mathrm{d}$ or $3 \mathrm{~g}$ fish oil $/ \mathrm{kg}$ body weight per $\mathrm{d}$. In order to provide the specified per $\mathrm{kg}$ body weight dose, the amount of EPA or fish oil in the diet was gradually increased until 4 months of age, the time at which the animals had reached a relatively stable adult body weight. At 4 months of age, the EPAsupplemented diet contained $21 \mathrm{mg} \mathrm{EPA} / \mathrm{g}$ of diet, and the fish oil-supplemented diet contained $13 \mathrm{mg}$ EPA plus $8 \mathrm{mg}$ DHA/g of diet. All the diets were supplemented with safflower oil to equalise the fat content of the three diets. The percentage energy from fat, protein and carbohydrate was 27,23 and $50 \%$, respectively. The fatty acid composition of the diet is shown in Table 1. Baseline body weights in all the groups were 110 (SD 2) g. Blood samples were collected from the tail after a $12 \mathrm{~h}$ fast each month up to 8 months of age for the measurement of glucose, insulin, TAG, NEFA, total cholesterol, adiponectin and leptin. Plasma CRP was measured at baseline and at 4 months of age.

\section{Assays}

Plasma glucose and cholesterol were measured using enzymatic colorimetric assays (Thermo DMA Louisville, CO, USA). Plasma TAG and NEFA were measured using enzymatic colorimetric assays (L-type TG H kit, NEFA Microtiter C kit; Wako Chemicals, Inc., Richmond, VA, USA). Plasma insulin, leptin and adiponectin were measured using rodent/rat-specific RIA (Millipore, St Charles, MO, USA). Plasma CRP was measured using ELISA (BD Biosciences, San Jose, CA, USA).

\section{Statistics and data analysis}

Data are presented as means with their standard errors. Statistical analyses were performed using GraphPad Prism 4.00 for Windows (GraphPad Software, San Diego, CA, USA).

Table 1. Experimental diet composition

\begin{tabular}{|c|c|c|c|}
\hline & Control & EPA & Fish oil \\
\hline Energy content (kJ/g diet) & $14 \cdot 6$ & $14 \cdot 6$ & $14 \cdot 6$ \\
\hline Safflower oil (g/kg diet) & 65 & 45 & - \\
\hline EPA (g/kg diet) & - & 20 & - \\
\hline Fish oil ( $\mathrm{g} / \mathrm{kg}$ diet) & - & - & 65 \\
\hline Crude fat ( $\mathrm{g} / \mathrm{kg}$ diet) & 117 & 117 & 117 \\
\hline \multicolumn{4}{|l|}{ Fatty acid (\% of total fatty acids) } \\
\hline 12:0 (Lauric) & 0.00 & 0.00 & 0.08 \\
\hline $14: 0$ (Myristic) & 0.46 & 0.46 & 4.82 \\
\hline 15: 0 (Pentadecanoic) & 0.00 & 0.00 & 0.32 \\
\hline $16: 0$ (Palmitic) & 10.09 & $9 \cdot 01$ & $16 \cdot 33$ \\
\hline $16: 1$ (Palmitoleic, $n-7$ ) & $0 \cdot 61$ & 0.61 & 5.57 \\
\hline 17: 0 (Heptadecanoic) & 0.00 & 0.00 & 0.27 \\
\hline 18:0 (Stearic) & 3.06 & $2 \cdot 62$ & 3.58 \\
\hline $18: 1$ (Oleic, $n-9$ ) & 17.59 & $15 \cdot 11$ & $16 \cdot 26$ \\
\hline $18: 2$ (Linoleic, $n-6)$ & 62.49 & $50 \cdot 32$ & 22.65 \\
\hline $18: 3(\gamma$ Linolenic, $n-6)$ & 0.00 & 0.00 & 0.17 \\
\hline $18: 3$ (Linolenic, $n-3)$ & $2 \cdot 42$ & $2 \cdot 42$ & 2.89 \\
\hline $18: 4$ (Octadecatetraenoic, $n-3$ ) & 0.09 & 0.09 & 2.02 \\
\hline $20: 0$ (Arachidic) & 0.15 & $0 \cdot 15$ & 0.33 \\
\hline $20: 1$ (Eicosenoic, $n-9)$ & 0.15 & 0.15 & 0.97 \\
\hline $20: 2$ (Eicosadienoic, $n-6$ ) & 0.04 & 0.04 & 0.17 \\
\hline $20: 4$ (Arachidonic, $n-6$ ) & 0.07 & 0.07 & 0.72 \\
\hline $20: 3$ (Eicosatrienoic, $n-3$ ) & 0.00 & 0.00 & 0.09 \\
\hline $20: 5$ (EPA, $n-3)$ & 0.59 & 17.95 & 11.09 \\
\hline $22: 0$ (Behenic) & 0.23 & 0.20 & 0.25 \\
\hline $22: 1$ (Erucic, $n-9$ ) & 0.00 & 0.00 & $0 \cdot 18$ \\
\hline $22: 5$ (Docosapentaenoic, $n-6$ ) & 0.11 & $0 \cdot 11$ & 1.41 \\
\hline 24: 0 (Lignoceric) & $0 \cdot 10$ & $0 \cdot 10$ & $0 \cdot 10$ \\
\hline $22: 6$ (DHA, $n-3)$ & 0.48 & 0.48 & $7 \cdot 11$ \\
\hline
\end{tabular}


Diabetes onset was analysed by log-rank testing of Kaplan-Meier survival curves up to 10 months of age. Longitudinal data were compared by two-factor repeatedmeasures ANOVA (time and treatment) followed by post hoc analysis with Bonferroni's multiple comparison test. One-factor ANOVA was used to analyse data assessed at a single time point. One animal from the control group and one animal from the fish oil-supplemented group became diabetic at too early an age to allow collection of blood samples up to 8 months of age and therefore were included in incidence analysis, but not in the longitudinal analyses. Differences were considered significant at $P<0 \cdot 05$.

\section{Results}

Effect of EPA and fish oil supplementation on type 2 diabetes mellitus onset, body weight and energy intake

There were no significant differences in the age of onset of diabetes or diabetes incidence between the three treatment groups (Fig. 1). Average ages of diabetes onset up to 1 year of age were 6.7 (SD 0.6 ), 6.0 (SD 0.6 ) and 6.0 (SD 0.8) months in the control, EPA-treated and fish oil-treated groups, respectively. Diabetes incidence was 85,87 and $88 \%$ in the control, EPA-treated and fish oil-treated groups, respectively. Energy intake did not differ significantly between treatment groups in the first 5 months of treatment (Fig. 2(a)). Starting at 6 months of age, fish oil-treated animals were consuming somewhat more energy than control animals $(P<0 \cdot 05)$. However, when prediabetic and diabetic animals were analysed separately, the average energy intake between 6 and 7 months of age did not differ among groups, and all diabetic animals consumed significantly more energy than prediabetic animals $(P<0 \cdot 001)$ (Fig. 2(b)). At 6 months of age, $56 \%$ of fish oil-treated animals were diabetic, whereas $26 \%$ of control animals were diabetic. Previously reported data demonstrate that UCD-T2DM rats develop progressive glucosuria and decreases in the circulating insulin and leptin concentrations after diabetes onset $^{(28)}$. Thus, following T2DM onset, animals increased

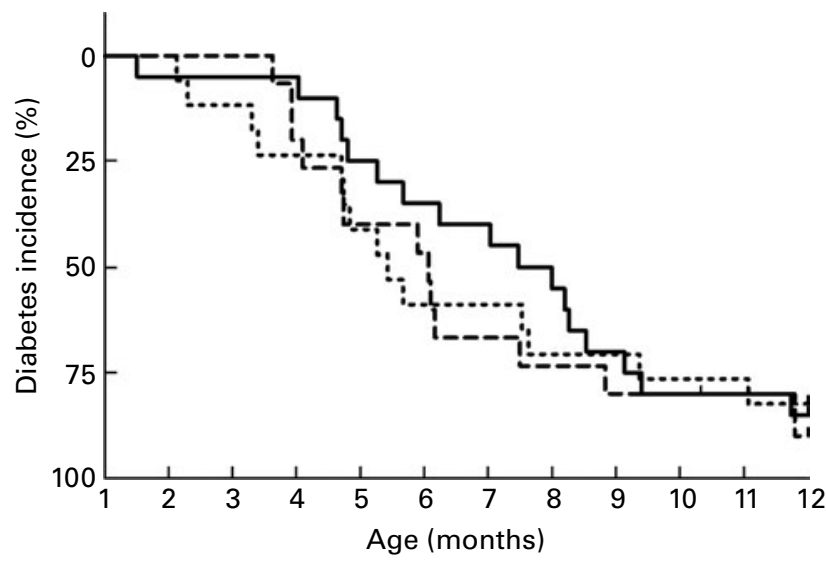

Fig. 1. Kaplan-Meier analysis of diabetes incidence in control ( $n$ 20), EPA-supplemented ( $n$ 15) and fish oil (FO)-supplemented ( $n$ 17) animals. Values were not significant by log-rank testing of Kaplan-Meier curves. - Control; =-_, EPA; ......, FO. their food intake to compensate for the energy lost from glucosuria, an effect that is probably mediated by the decreases in insulin and leptin ${ }^{(28)}$. There were no differences in body weight between treatment groups (Fig. 2(c)).
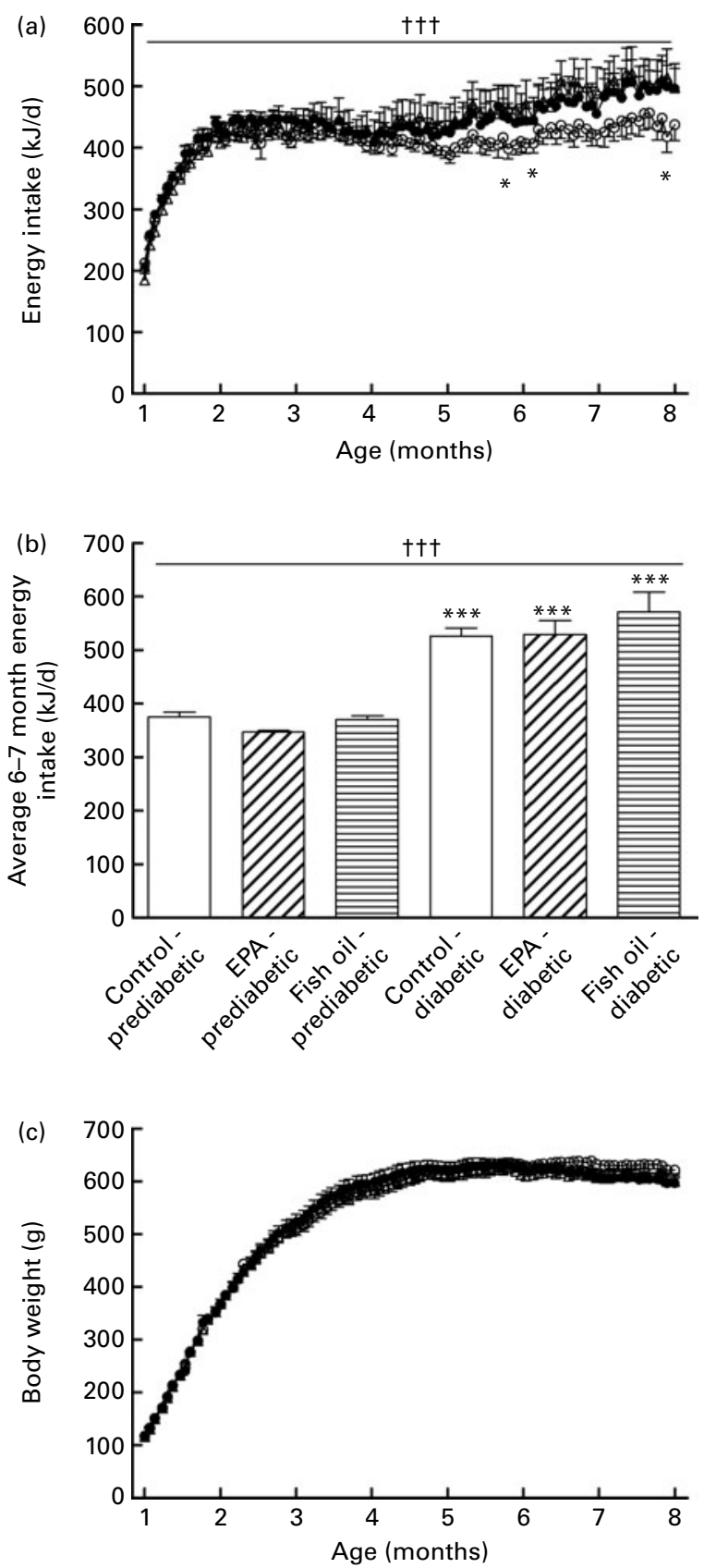

Fig. 2. Energy intake (metabolisable energy) (a) and body weight (c) in control ( $n$ 19), EPA-supplemented ( $n$ 15) and fish oil (FO)-supplemented ( $n$ 16) animals. ††† Mean values were compared using two-factor (time and treatment) repeated-measures ANOVA $(P<0.001)$. ${ }^{*}$ Mean values were significantly different when compared with fish oil by Bonferroni's post test $(P<0.05)$. Average energy intake (b) between 6 and 7 months of age in prediabetic control ( $n$ 13), EPA-supplemented $(n 5)$ and fish oil-supplemented ( $n$ 6) animals, and diabetic control ( $n$ 6), EPA-supplemented ( $n 10)$ and fish oil-supplemented ( $n$ 10) animals. †††Mean values were compared using one-factor ANOVA $(P<0.0001)$. ${ }^{* \star *}$ Mean values were significantly different when compared with values in prediabetic animals by Bonferroni's post test $(P<0.001)$. - O-, Control; - - - EPA; $\triangle$, FO. 
Effect of EPA and fish oil supplementation on fasting plasma glucose, lipids, hormones and $C$-reactive protein

Fasting plasma glucose concentrations did not differ between groups (Fig. 3(a)). However, fasting plasma glucose concentrations tended to increase in the fish oil-supplemented and EPA-supplemented groups starting at 7 months of age. This trend was due to the higher incidence of diabetes in the fish oil-treated and EPA-treated groups compared with the control group. At 7 months of age, $37 \%$ of control animals were diabetic, whereas 67 and $56 \%$ of EPA-supplemented and fish oil-supplemented animals were diabetic, respectively. Fasting plasma insulin concentrations did not differ between groups; however, animals supplemented with fish oil tended to have lower fasting plasma insulin concentrations than control animals after 4 months of treatment (Fig. 3(b)).

Fish oil supplementation lowered fasting plasma TAG concentrations compared with control (-39 (SD 7) \% at 4 months of age) (Fig. 4(a)). Overall, EPA supplementation did not significantly lower fasting plasma TAG concentrations compared with control animals. However, EPA supplementation lowered fasting plasma TAG concentrations in diabetic animals $(-45$ (SD 3) \%) at 4 months of age compared with 4-month-old diabetic control animals ( $P<0 \cdot 001$, control: $n$ 4, fish oil: $n 6)$. EPA supplementation lowered fasting plasma NEFA concentrations by 23 (SD 5) \% compared with control at 4 months of age $(P<0.05)($ Fig. 4(b)). Fish oil supplementation significantly
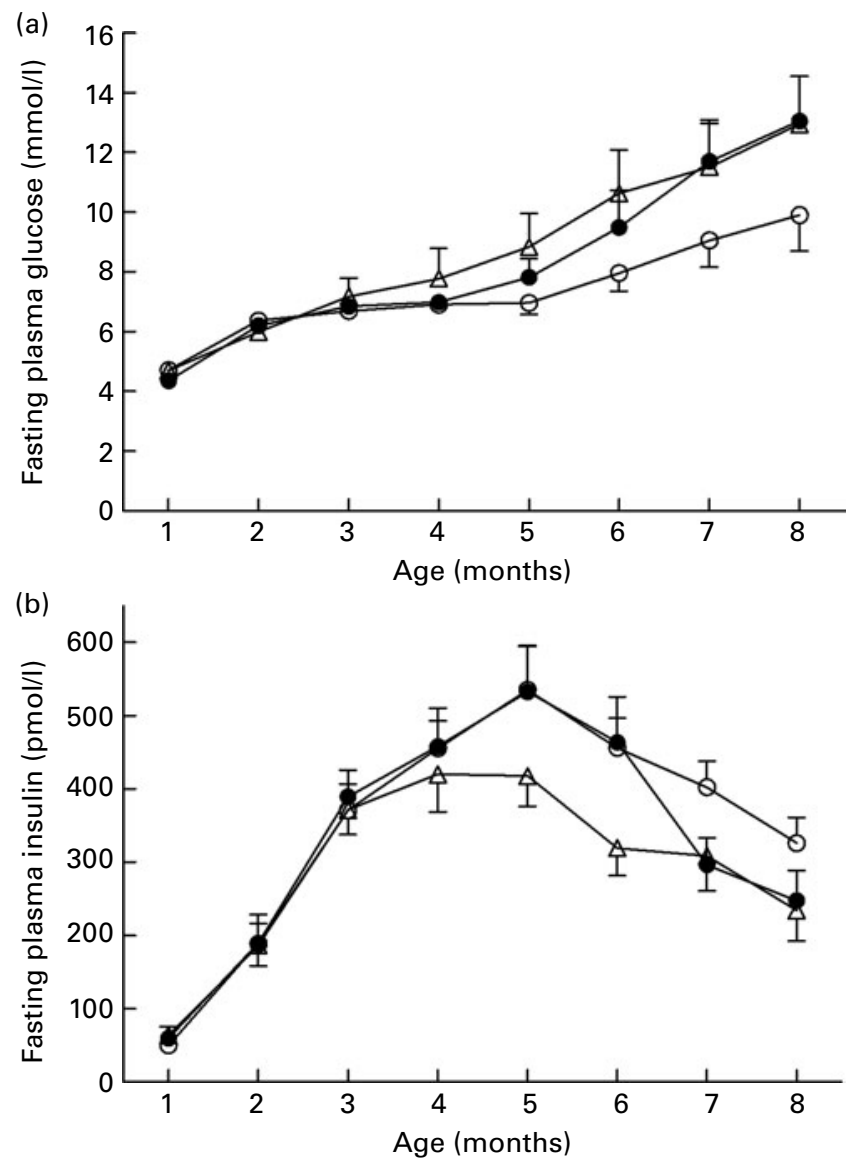

Fig. 3. Fasting plasma glucose (a) and insulin (b) concentrations in control ( $n$ 19), EPA-supplemented ( $n$ 15) and fish oil (FO)-supplemented ( $n$ 16) animals. -O- Control; -๑-, EPA; $\triangle$, FO.
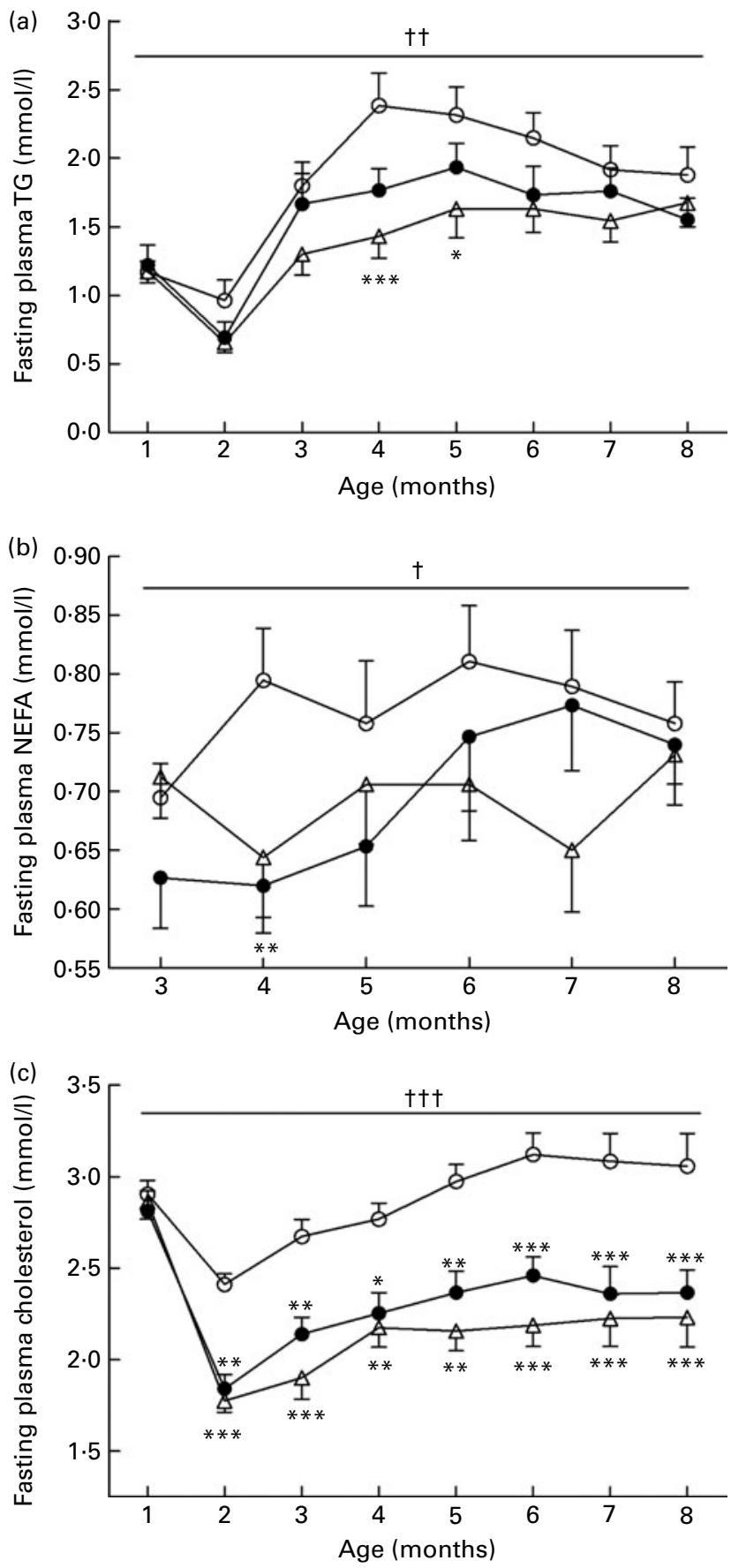

Fig. 4. Fasting plasma TAG (a), NEFA (b) and cholesterol (c) concentrations in control ( $n$ 19), EPA-supplemented ( $n$ 15) and fish oil (FO)-supplemented ( $n$ 16) animals. Mean values were significantly different by two-factor ANOVA: $\dagger P<0.05, \dagger \dagger P<0.01, \dagger+\uparrow<0.001$. Mean values were significantly different from those of the control group by Bonferroni's post test: ${ }^{\star} P<0.05$, ${ }^{\star \star} P<0.01,{ }^{\star \star \star} P<0.001$. $-O-$, Control; - - - EPA; $\triangle$, FO.

lowered fasting plasma cholesterol concentrations throughout the sampling period ( -22 (SD 4$) \%$ at 4 months of age) (Fig. 4(c)). EPA supplementation also lowered fasting plasma cholesterol concentrations ( -19 (SD 4$) \%$ at 4 months of age).

Fasting plasma adiponectin and leptin concentrations did not differ between groups over the course of the study (Fig. 5(a) and (b)). Fasting plasma CRP concentrations did not differ between groups at 4 months of age (Table 2). 

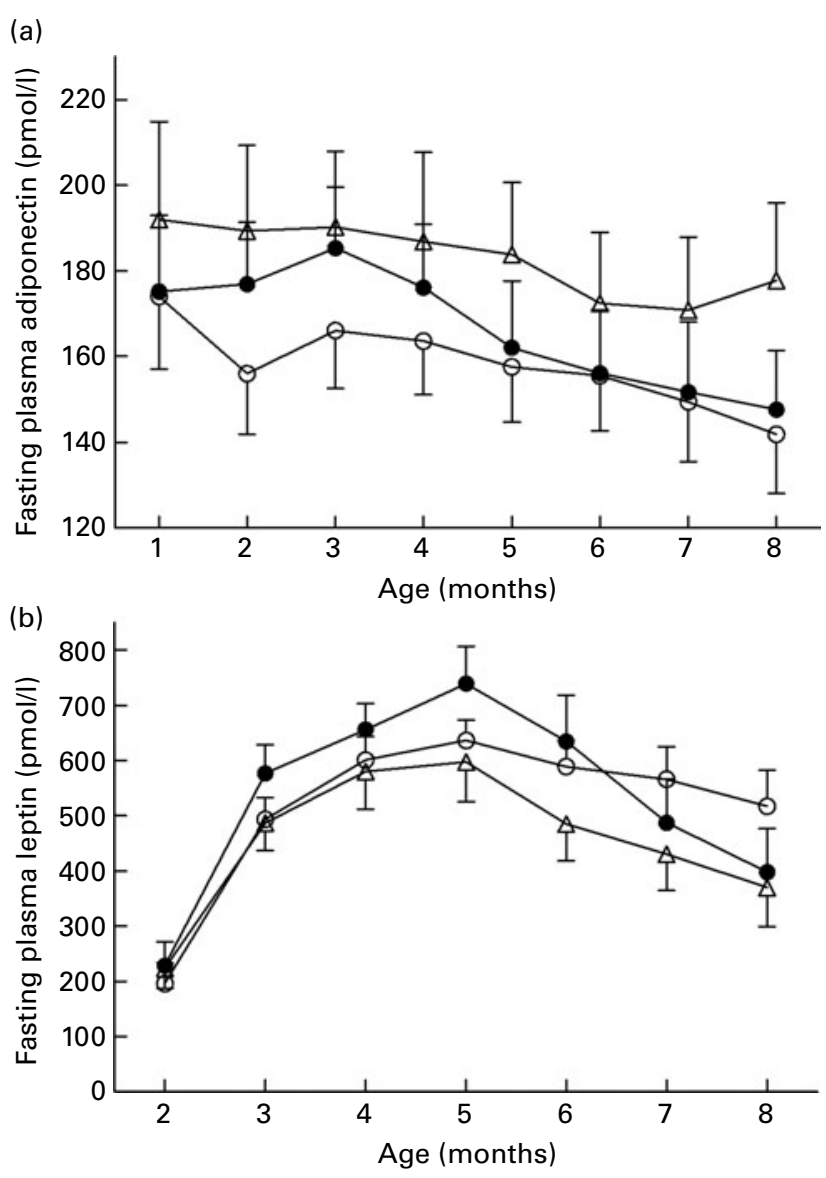

Fig. 5. Fasting plasma adiponectin (a) and leptin (b) concentrations in control ( $n$ 19), EPA-supplemented $(n 15)$ and fish oil (FO)-supplemented $(n 16)$ animals. -O-, Control; -๑-, EPA; $\triangle$, FO.

\section{Discussion}

Previous studies investigating the effects of EPA and fish oil have demonstrated the anti-inflammatory ${ }^{(1,33)}$ and cardioprotective properties for these supplements ${ }^{(6-9)}$. However, evidence that fish oil or EPA increases insulin sensitivity and improves glucose tolerance has been conflicting ${ }^{(7,18,25,27)}$. Results from the present study indicate that while chronic supplementation with EPA ( $1 \mathrm{~g} / \mathrm{kg}$ body weight per $\mathrm{d})$ or fish oil ( $3 \mathrm{~g} / \mathrm{kg}$ body weight per $\mathrm{d}$ ) for more than 8 months lowers circulating lipids (TAG, NEFA and cholesterol), neither treatment delays the onset of diabetes in UCD-T2DM rats.

Similar to previous results from studies in animals and in human subjects, energy intake, body weight and circulating leptin were not affected by EPA or fish oil supplementation $^{(4,6,23,27,34-36)}$. In some rodent studies, decreases in

Table 2. Plasma C-reactive protein concentrations

(Mean values with their standard errors)

\begin{tabular}{lcccccc}
\hline & \multicolumn{2}{c}{1 month $(\mathrm{nmol} / \mathrm{l})$} & & \multicolumn{2}{c}{4 months $(\mathrm{nmol} / \mathrm{l})$} & \\
\cline { 2 - 3 } & Mean & SEM & & Mean & SEM & $n$ \\
\hline Control & 295 & 42 & & 604 & 91 & 19 \\
EPA & 228 & 20 & & 560 & 60 & 15 \\
Fish oil & 231 & 23 & & 527 & 57 & 16 \\
\hline
\end{tabular}

body weight have been reported during EPA and fish oil treatment, which the authors attributed, in part, to increases in circulating leptin ${ }^{(5,11)}$. However, these results have not been replicated in a number of other studies in rodents or human subjects ${ }^{(4,6,23,27,34-36)}$.

Neither EPA nor fish oil supplementation affected fasting plasma glucose or insulin concentrations, suggesting that these supplements did not affect insulin sensitivity in the UCD-T2DM rat model of diabetes. These findings are in agreement with previous studies in rodents ${ }^{(23,36)}$ and several clinical studies in patients with T2DM, in which no improvements of glycaemic control, circulating insulin or insulin sensitivity were observed during treatment with fish oil ${ }^{(25,37)}$. In some clinical studies, insulin sensitivity and glycaemic control actually worsened during fish oil treatment, despite marked improvements in circulating lipids ${ }^{(7,38-40)}$. One possible explanation for a lack of improvement of glycaemic control with EPA or fish oil supplementation is that EPA and fish oil may increase hepatic glucose output by increasing hepatic free fatty acid oxidation and decreasing hepatic glucose oxidation, as would be predicted by changes in the Randle glucose-fatty acid cycle ${ }^{(41)}$. This is supported by the data generated by Holness et al. ${ }^{(23)}$, reporting decreased insulinmediated suppression of endogenous glucose production and impaired glucose-stimulated insulin secretion in animals fed a diet supplemented with EPA and DHA. Furthermore, Mostad et al. ${ }^{(27)}$ reported decreased glucose utilisation, measured with euglycaemic clamps, in patients with T2DM supplemented with fish oil.

Both EPA and fish oil supplementation substantially lowered circulating lipids in prediabetic and diabetic animals. $n-3$ Fatty acids have been suggested to lower circulating lipids by their interactions with several nuclear receptors, including the liver X receptor, hepatocyte nuclear factor- $4 \alpha$, farnesol X receptor and the PPAR ${ }^{(8)} \cdot n-3$ Fatty acids have been shown to decrease de novo lipogenesis through inhibition of sterolregulatory-element-binding protein- $1 \mathrm{c}^{(42)}$ and to increase fatty acid oxidation in liver and muscle through activation of PPAR- $\alpha^{(43,44)}$ and by down-regulating hepatocyte nuclear factor- $4 \alpha$, resulting in a increased flux of glucose to glycogen ${ }^{(45)}$. Together, these effects favour fatty acid oxidation over fatty acid storage as TAG. Interestingly, it appeared that fish oil more potently lowered circulating TAG than did EPA in prediabetic animals. The differential effect of EPA and fish oil treatment on circulating lipids in UCD-T2DM rats is supported by the previous studies in which the TAG-lowering effect of DHA (present in fish oil) was reported to be greater than that of EPA in healthy and hyperlipidaemic men ${ }^{(6,18)}$. The transient nature of the decreases in circulating NEFA and TAG with EPA and fish oil treatment can be attributed to increasing diabetes incidence at older ages, as we have previously reported that fasting plasma TAG decreases and fasting plasma NEFA increases after diabetes onset in the UCD-T2DM rat ${ }^{(28)}$.

Fatty acids in fish oil, such as EPA and DHA, have been shown to activate PPAR- $\gamma$ resulting in increased adiponectin production and secretion ${ }^{(20)}$. Adiponectin is secreted from adipocytes and promotes insulin sensitivity, in part, by stimulating the oxidation of ectopic TAG via activation of adenosine monophosphate-activated protein kinase in liver and skeletal muscle ${ }^{(19,46)}$. However, we did not observe a significant 
increase in circulating adiponectin concentrations during EPA or fish oil supplementation, similar to previous human studies $^{(47,48)}$. Thus, increased adiponectin production does not appear to contribute to the lipid-lowering effects of EPA and fish oil treatment observed in the present study.

While plasma CRP concentrations were slightly (approximately 8-12\%) lower in EPA-supplemented and fish oil-supplemented animals, this trend did not reach significance, suggesting that EPA and fish oil do not effectively reduce inflammation in UCD-T2DM rats, or that higher doses may be required. Interestingly, there have been conflicting reports on the effect of EPA or fish oil treatment on markers of inflammation in individuals with diabetes, suggesting that the effects of these supplements to reduce inflammation may be at least partially dependent on metabolic status and thus requires further investigation ${ }^{(3,4,49)}$. This lack of an anti-inflammatory effect may also, in part, explain the lack of effect of EPA or fish oil supplementation to delay diabetes onset, as inflammation is known to promote the development of insulin resistance and diabetes ${ }^{(16,17)}$.

Despite the widespread use of both fish oil and EPA supplements, this is the first study to investigate the potential for EPA and fish oil to delay or prevent the development of T2DM in the UCD-T2DM rat model of type 2 diabetes. The data indicate that EPA and fish oil supplementation do not delay diabetes onset, nor do they affect body weight, plasma glucose or insulin concentrations. Thus, these results do not provide evidence to support the use of EPA and fish oil for the prevention of T2DM. However, the results do confirm that EPA and fish oil are useful in the management of hyperlipidaemia, a common co-morbidity of T2DM.

\section{Acknowledgements}

We thank Hong-Duc Ta and Sunhye Kim for their extensive help with animal care and monitoring. We would also like to thank Susan Bennett, Cheryl Phillips and the Meyer Hall Animal Facility for excellent animal care. B. P. C., K. L. S., J. L. G., S. C. G. and P. J. H. developed and characterised the UCD-T2DM rat model used for the study. K. L. S., J. L. G., S. C. G. and P. J. H. designed and obtained funding for the study; B. P. C., K. L. S. and J. L. G. conducted the experiments; B. P. C., K. L. S., J. L. G. and P. J. H. analysed the data; B. P. C., K. L. S. and P. J. H. wrote the manuscript; B. P. C. and P. J. H. had primary responsibility for final content. All authors read and approved the final manuscript. The present project was supported by a National Institutes of Health (NIH) grant (AT002993). Dr Havel's laboratory also receives support from NIH grants (R01 HL075675, R01 HL091333, AT002599, AT003645) and the American Diabetes Association. B. P. C., K. L. S., J. L. G., S. C. G. and P. J. H. have no conflicts of interest.

\section{References}

1. Calder PC (2002) Dietary modification of inflammation with lipids. Proc Nutr Soc 61, 345-358.

2. Li H, Ruan XZ, Powis SH, et al. (2005) EPA and DHA reduce LPS-induced inflammation responses in HK-2 cells: evidence for a PPAR-gamma-dependent mechanism. Kidney Int 67, $867-874$.
3. Itoh M, Suganami T, Satoh N, et al. (2007) Increased adiponectin secretion by highly purified eicosapentaenoic acid in rodent models of obesity and human obese subjects. Arterioscler Thromb Vasc Biol 27, 1918-1925.

4. Kabir M, Skurnik G, Naour N, et al. (2007) Treatment for 2 mo with $n 3$ polyunsaturated fatty acids reduces adiposity and some atherogenic factors but does not improve insulin sensitivity in women with type 2 diabetes: a randomized controlled study. Am J Clin Nutr 86, 1670-1679.

5. Perez-Matute P, Perez-Echarri N, Martinez JA, et al. (2007) Eicosapentaenoic acid actions on adiposity and insulin resistance in control and high-fat-fed rats: role of apoptosis, adiponectin and tumour necrosis factor-alpha. Br J Nutr 97, 389-398.

6. Grimsgaard S, Bonaa KH, Hansen JB, et al. (1997) Highly purified eicosapentaenoic acid and docosahexaenoic acid in humans have similar triacylglycerol-lowering effects but divergent effects on serum fatty acids. Am J Clin Nutr 66, 649-659.

7. Woodman RJ, Mori TA, Burke V, et al. (2002) Effects of purified eicosapentaenoic and docosahexaenoic acids on glycemic control, blood pressure, and serum lipids in type 2 diabetic patients with treated hypertension. Am J Clin Nutr 76, 1007-1015.

8. Davidson MH (2006) Mechanisms for the hypotriglyceridemic effect of marine omega-3 fatty acids. Am J Cardiol 98, 27i-33i.

9. Yokoyama M, Origasa H, Matsuzaki M, et al. (2007) Effects of eicosapentaenoic acid on major coronary events in hypercholesterolaemic patients (JELIS): a randomised open-label, blinded endpoint analysis. Lancet 369, 1090-1098.

10. Storlien LH, Baur LA, Kriketos AD, et al. (1996) Dietary fats and insulin action. Diabetologia 39, 621-631.

11. Rossi AS, Lombardo YB, Lacorte JM, et al. (2005) Dietary fish oil positively regulates plasma leptin and adiponectin levels in sucrose-fed, insulin-resistant rats. Am J Physiol Regul Integr Comp Physiol 289, R486-R494.

12. Jump DB (2002) The biochemistry of $n-3$ polyunsaturated fatty acids. J Biol Chem 277, 8755-8758.

13. Plutzky J (2003) The potential role of peroxisome proliferatoractivated receptors on inflammation in type 2 diabetes mellitus and atherosclerosis. Am J Cardiol 92, 34J-41J.

14. Zhao Y, Joshi-Barve S, Barve S, et al. (2004) Eicosapentaenoic acid prevents LPS-induced TNF-alpha expression by preventing NF-kappaB activation. J Am Coll Nutr 23, 71-78.

15. Ziouzenkova O \& Plutzky J (2004) Lipolytic PPAR activation: new insights into the intersection of triglycerides and inflammation? Curr Opin Clin Nutr Metab Care 7, 369-375.

16. Yudkin JS, Stehouwer CD, Emeis JJ, et al. (1999) C-reactive protein in healthy subjects: associations with obesity, insulin resistance, and endothelial dysfunction: a potential role for cytokines originating from adipose tissue? Arterioscler Thromb Vasc Biol 19, 972-978.

17. Tataranni PA \& Ortega E (2005) A burning question: does an adipokine-induced activation of the immune system mediate the effect of overnutrition on type 2 diabetes? Diabetes $\mathbf{5 4}$, 917-927.

18. Mori TA, Burke V, Puddey IB, et al. (2000) Purified eicosapentaenoic and docosahexaenoic acids have differential effects on serum lipids and lipoproteins, LDL particle size, glucose, and insulin in mildly hyperlipidemic men. Am J Clin Nutr 71, 1085-1094.

19. Havel PJ (2004) Update on adipocyte hormones: regulation of energy balance and carbohydrate/lipid metabolism. Diabetes 53, Suppl. 1, S143-S151.

20. Neschen S, Morino K, Rossbacher JC, et al. (2006) Fish oil regulates adiponectin secretion by a peroxisome proliferator-activated receptor-gamma-dependent mechanism in mice. Diabetes 55, 924-928.

21. Semple RK, Chatterjee VK \& O'Rahilly S (2006) PPAR gamma and human metabolic disease. J Clin Invest 116, 581-589. 
22. D'Alessandro ME, Lombardo YB \& Chicco A (2002) Effect of dietary fish oil on insulin sensitivity and metabolic fate of glucose in the skeletal muscle of normal rats. Ann Nutr Metab 46, 114-120.

23. Holness MJ, Greenwood GK, Smith ND, et al. (2003) Diabetogenic impact of long-chain omega-3 fatty acids on pancreatic beta-cell function and the regulation of endogenous glucose production. Endocrinology 144, 3958-3968.

24. Holness MJ, Smith ND, Greenwood GK, et al. (2004) Acute omega-3 fatty acid enrichment selectively reverses high-saturated fat feeding-induced insulin hypersecretion but does not improve peripheral insulin resistance. Diabetes 53, Suppl. 1, S166-S171.

25. Montori VM, Farmer A, Wollan PC, et al. (2000) Fish oil supplementation in type 2 diabetes: a quantitative systematic review. Diabetes Care 23, 1407-1415.

26. Faeh D, Minehira K, Schwarz JM, et al. (2005) Effect of fructose overfeeding and fish oil administration on hepatic de novo lipogenesis and insulin sensitivity in healthy men. Diabetes $\mathbf{5 4}$, 1907-1913.

27. Mostad IL, Bjerve KS, Bjorgaas MR, et al. (2006) Effects of $n-3$ fatty acids in subjects with type 2 diabetes: reduction of insulin sensitivity and time-dependent alteration from carbohydrate to fat oxidation. Am J Clin Nutr 84, 540-550.

28. Cummings BP, Digitale EK, Stanhope KL, et al. (2008) Development and characterization of a novel rat model of type 2 diabetes mellitus: the UC Davis type 2 diabetes mellitus UCD-T2DM rat. Am J Physiol Regul Integr Comp Physiol 295, R1782-R1793

29. Stanhope KL, Kras KM \& Moreno-Aliaga MJ (2000) A comparison of adipocyte size and metabolism in Charles River and Harlan Sprague Dawley rats (Abstract). Obes Res 8, 66S.

30. Stanhope KL, Sinha M, Graham J, et al. (2002) Low circulating adiponectin levels and reduced adipocyte adiponectin production in obese, insulin-resistant Sprague-Dawley rats (Abstract). Diabetes 52, A404.

31. Griffen SC, Wang J \& German MS (2001) A genetic defect in beta-cell gene expression segregates independently from the fa locus in the ZDF rat. Diabetes 50, 63-68.

32. Bergeron R, Yao J, Woods JW, et al. (2006) Peroxisome proliferator-activated receptor (PPAR)-alpha agonism prevents the onset of type 2 diabetes in Zucker diabetic fatty rats: a comparison with PPAR gamma agonism. Endocrinology 147, 4252-4262.

33. Yaqoob P \& Calder P (1995) Effects of dietary lipid manipulation upon inflammatory mediator production by murine macrophages. Cell Immunol 163, 120-128.

34. Raclot T, Groscolas R, Langin D, et al. (1997) Site-specific regulation of gene expression by $n-3$ polyunsaturated fatty acids in rat white adipose tissues. J Lipid Res 38, 1963-1972.

35. Reseland JE, Haugen F, Hollung K, et al. (2001) Reduction of leptin gene expression by dietary polyunsaturated fatty acids. J Lipid Res 42, 743-750.
36. Neschen S, Morino K, Dong J, et al. (2007) n-3 Fatty acids preserve insulin sensitivity in vivo in a peroxisome proliferatoractivated receptor-alpha-dependent manner. Diabetes 56, 1034-1041.

37. Annuzzi G, Rivellese A, Capaldo B, et al. (1991) A controlled study on the effects of $n-3$ fatty acids on lipid and glucose metabolism in non-insulin-dependent diabetic patients. Atherosclerosis 87, 65-73.

38. Vessby B \& Boberg M (1990) Dietary supplementation with $n-3$ fatty acids may impair glucose homeostasis in patients with non-insulin-dependent diabetes mellitus. J Intern Med 228, $165-171$.

39. Vessby B, Karlstrom B, Boberg M, et al. (1992) Polyunsaturated fatty acids may impair blood glucose control in type 2 diabetic patients. Diabet Med 9, 126-133.

40. Dunstan DW, Mori TA, Puddey IB, et al. (1997) The independent and combined effects of aerobic exercise and dietary fish intake on serum lipids and glycemic control in NIDDM. A randomized controlled study. Diabetes Care 20, 913-921.

41. Randle PJ (1998) Regulatory interactions between lipids and carbohydrates: the glucose fatty acid cycle after 35 years. Diabetes Metab Rev 14, 263-283.

42. Pawar A, Botolin D, Mangelsdorf DJ, et al. (2003) The role of liver $\mathrm{X}$ receptor-alpha in the fatty acid regulation of hepatic gene expression. J Biol Chem 278, 40736-40743.

43. Berge RK, Madsen L, Vaagenes H, et al. (1999) In contrast with docosahexaenoic acid, eicosapentaenoic acid and hypolipidaemic derivatives decrease hepatic synthesis and secretion of Triacylglycerol by decreased diacylglycerol acyltransferase activity and stimulation of fatty acid oxidation. Biochem $J$ Pt 1, 343, 191-197.

44. Blaschke F, Takata Y, Caglayan E, et al. (2006) Obesity, peroxisome proliferator-activated receptor, and atherosclerosis in type 2 diabetes. Arterioscler Thromb Vasc Biol 26, 28-40.

45. Pegorier JP, Le May C \& Girard J (2004) Control of gene expression by fatty acids. J Nutr 134, 2444S-2449S.

46. Long YC \& Zierath JR (2006) AMP-activated protein kinase signaling in metabolic regulation. $J$ Clin Invest 116, $1776-1783$.

47. Lara JJ, Economou M, Wallace AM, et al. (2007) Benefits of salmon eating on traditional and novel vascular risk factors in young, non-obese healthy subjects. Atherosclerosis 193, 213-221.

48. Kratz M, Swarbrick MM, Callahan HS, et al. (2008) Effect of dietary $n-3$ polyunsaturated fatty acids on plasma total and high-molecular-weight adiponectin concentrations in overweight to moderately obese men and women. Am J Clin Nutr 87, 347-353.

49. Gillam M, Noto A, Zahradka P, et al. (2009) Improved $n-3$ fatty acid status does not modulate insulin resistance in fa/fa Zucker rats. Prostaglandins Leukot Essent Fatty Acids 81, 331-339. 